the full article

entitled

\title{
Tetronamides as Latent Acyclic Vinylogous Amides in Formal Aza-[3 + 3] Cycloaddition Reactions with ๑,,- Unsaturated Iminium Salts. An Unexpected Rearrangement and An Approach to Synthesis of Substituted Piperidines.
}

\author{
authored by
}

Nadiya Sydorenko, Richard P. Hsung*, Ossama Saleh Darwish, Juliet M. Hahn, and Jia Liu

Department of Chemistry, University of Minnesota, Minneapolis, MN 55455

X-ray Structural Report For 35c.

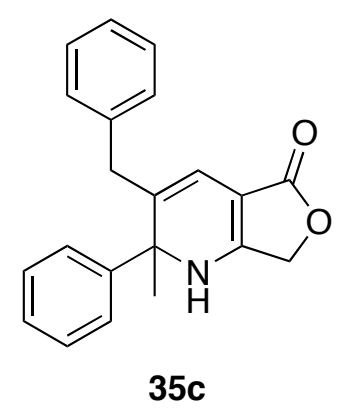


REFERENCE NUMBER: 03139

CRYSTAL STRUCTURE REPORT

$$
\mathrm{C}_{21} \mathrm{H}_{19} \mathrm{~N} \mathrm{O}_{2}
$$

Report prepared for:

N. Sydorenko, Prof. R. Hsung

July 12, 2003

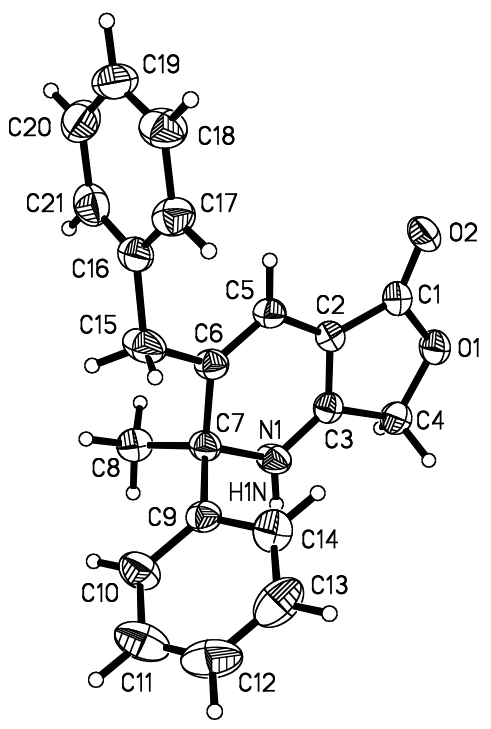

William W. Brennessel

X-Ray Crystallographic Laboratory

Department of Chemistry

University of Minnesota

207 Pleasant St. S.E.

Minneapolis, MN 55455 


\section{Data collection}

A crystal (approximate dimensions $0.45 \times 0.32 \times 0.24 \mathrm{~mm}^{3}$ ) was placed onto the tip of a $0.1 \mathrm{~mm}$ diameter glass capillary and mounted on a Bruker SMART Platform CCD diffractometer for a data collection at 173(2) K. A preliminary set of cell constants was calculated from reflections harvested from three sets of 20 frames. These initial sets of frames were oriented such that orthogonal wedges of reciprocal space were surveyed. This produced initial orientation matrices determined from 108 reflections. The data collection was carried out using MoK $\square$ radiation (graphite monochromator) with a frame time of 25 seconds and a detector distance of $4.94 \mathrm{~cm}$. A randomly oriented region of reciprocal space was surveyed to the extent of one sphere and to a resolution of $0.84 \AA$. Four major sections of frames were collected with $0.30^{\circ}$ steps in $\square$ at four different $\square$ settings and a detector position of $-28^{\circ}$ in $2 \square$. The intensity data were corrected for absorption and decay (SADABS). ${ }^{1}$ Final cell constants were calculated from the xyz centroids of 952 strong reflections from the actual data collection after integration (SAINT). ${ }^{2}$ Please refer to Table 1 for additional crystal and refinement information.

\section{Structure solution and refinement}

The structure was solved using SHELXS-9 $7^{3}$ and refined using SHELXL-97. ${ }^{3}$ The space group $P 2_{1} / c$ was determined based on systematic absences and intensity statistics. A direct-methods solution was calculated which provided most non-hydrogen atoms from the E-map. Full-matrix least squares / difference Fourier cycles were performed which located the remaining non-hydrogen atoms. All non-hydrogen atoms were refined with anisotropic displacement parameters. All hydrogen atoms except for $\mathrm{H} 1 \mathrm{~N}$ and $\mathrm{H} 2 \mathrm{~N}$ were placed in ideal positions and refined as riding atoms with relative isotropic displacement parameters. The hydrogen atoms bound to the nitrogen atoms were refined positionally independent of the nitrogen atoms (see Table 7 for intermolecular hydrogen bonding information). The final full matrix least squares refinement converged to $R 1=0.0368$ and $w R 2=0.1004$ ( $F^{2}$, all data).

\section{Structure description}

The structure is the one suggested. There are two independent molecules in the asymmetric unit. The molecule containing $\mathrm{N} 2$ is disordered in the phenyl and benzyl portions (50:50 as refined for both).

Data collection and structure solution were conducted at the X-Ray Crystallographic Laboratory, 160 Kolthoff Hall, Department of Chemistry, University of Minnesota. All calculations were performed using Pentium computers using the current SHELXTL suite of programs. All publications arising from this report MUST either 1)include William W. Brennessel as a coauthor or 2)acknowledge William W. Brennessel, Victor G. Young, Jr., and the XRay Crystallographic Laboratory. 
1 An empirical correction for absorption anisotropy, R. Blessing, Acta Cryst. A51, 33-38 (1995).

2 SAINT V6.2, Bruker Analytical X-Ray Systems, Madison, WI (2001).

3 SHELXTL V6.10, Bruker Analytical X-Ray Systems, Madison, WI (2000).

$$
\begin{gathered}
\text { Some equations of interest: } \\
R_{\text {int }}=\square\left|F_{\mathrm{o}}^{2}-<F_{\mathrm{o}}{ }^{2}>\right| / \square\left|F_{\mathrm{o}}{ }^{2}\right| \\
R_{1}=\square|| F_{\mathrm{o}}|-| F_{\mathrm{c}} \| / \square\left|F_{\mathrm{o}}\right| \\
w R 2=\left[\square\left[w\left(F_{\mathrm{o}}{ }^{2}-F_{\mathrm{c}}{ }^{2}\right)^{2}\right] / \square\left[w\left(F_{\mathrm{o}}{ }^{2}\right)^{2}\right]\right]^{1 / 2} \\
\text { where } w=q /\left[\square^{2}\left(F_{\mathrm{o}}{ }^{2}\right)+\left(a^{*} P\right)^{2}+b^{*} P+d+e^{*} \sin (\square)\right] \\
\mathrm{GooF}=S=\left[\square\left[w\left(F_{\mathrm{o}}{ }^{2}-F_{\mathrm{c}}{ }^{2}\right)^{2}\right] /(n-p)\right]^{1 / 2}
\end{gathered}
$$



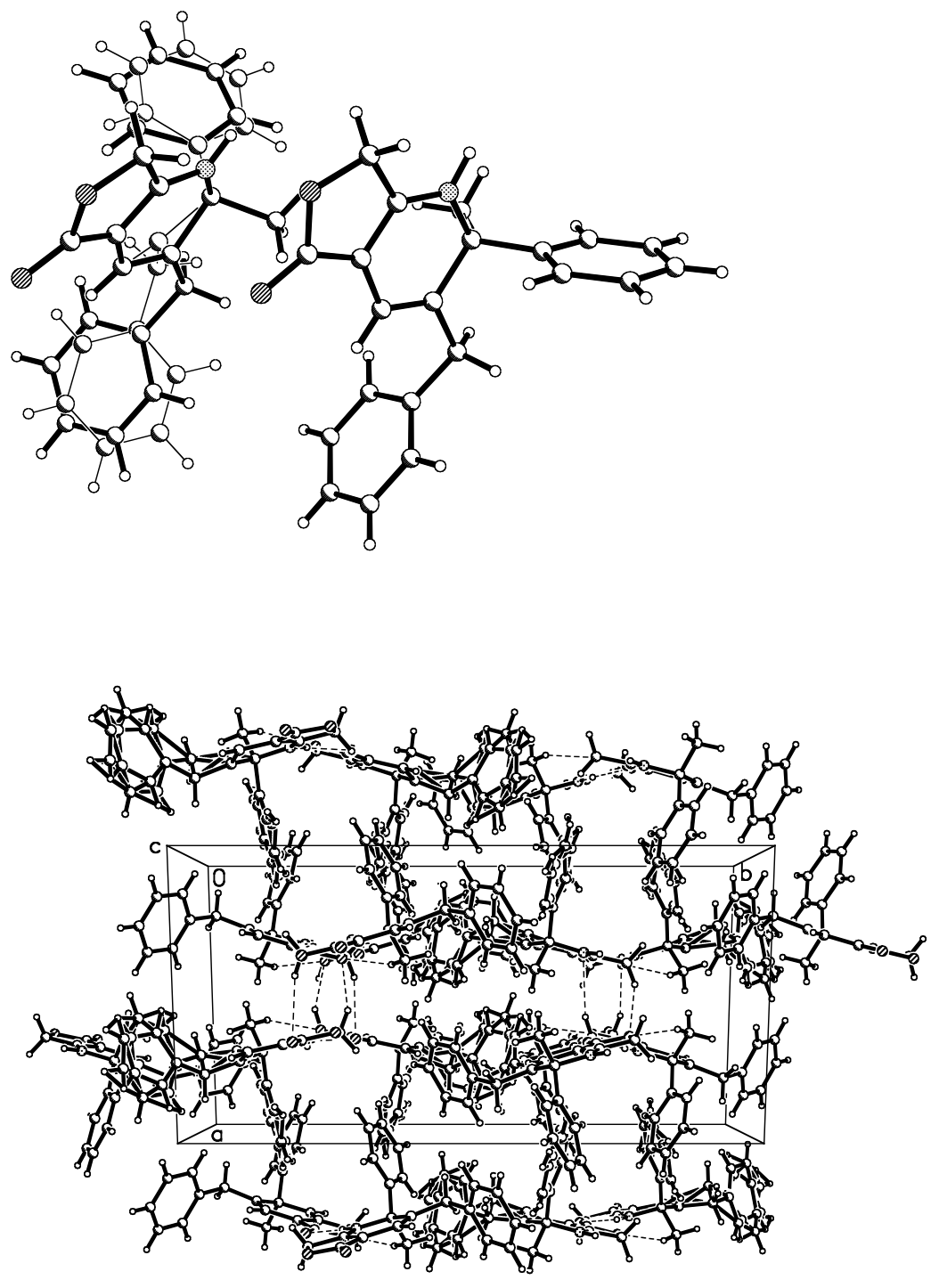

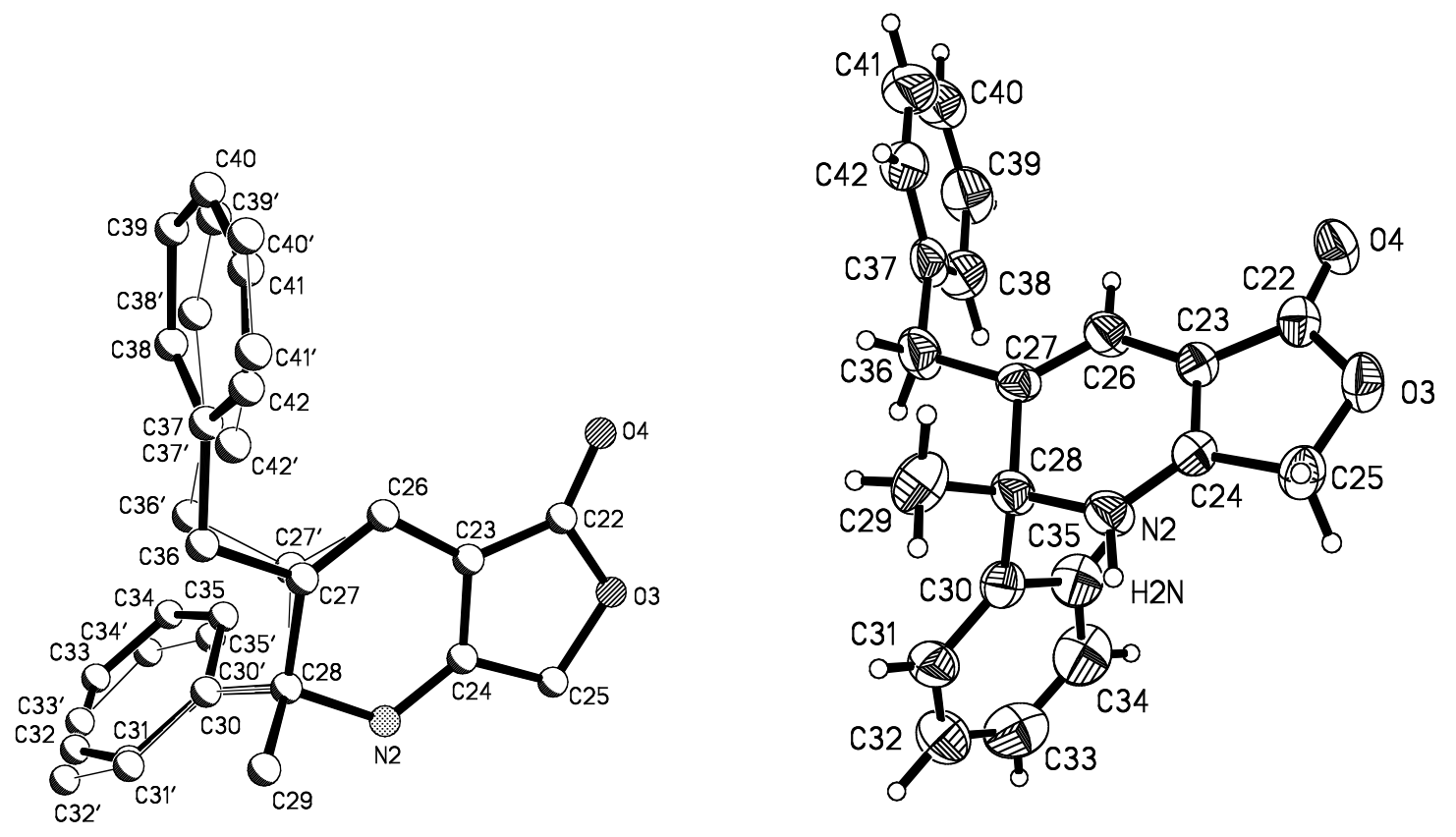
Table 1. Crystal data and structure refinement for 03139.

\begin{tabular}{|c|c|c|}
\hline Identification code & \multicolumn{2}{|l|}{03139} \\
\hline Empirical formula & \multicolumn{2}{|l|}{ C21 H19 N O2 } \\
\hline Formula weight & \multicolumn{2}{|l|}{317.37} \\
\hline Temperature & \multicolumn{2}{|l|}{$173(2) \mathrm{K}$} \\
\hline Wavelength & \multicolumn{2}{|l|}{$0.71073 \AA$} \\
\hline Crystal system & \multicolumn{2}{|l|}{ Monoclinic } \\
\hline Space group & \multicolumn{2}{|l|}{$P 2_{1} / c$} \\
\hline \multirow[t]{3}{*}{ Unit cell dimensions } & $a=11.906(2) \AA$ & $\square=90^{\circ}$ \\
\hline & $b=23.016(4) \AA$ & $\square=104.836(3)^{\circ}$ \\
\hline & $c=12.888(2) \AA$ & $\square=90^{\circ}$ \\
\hline Volume & \multicolumn{2}{|l|}{$3414.1(9) \AA^{3}$} \\
\hline$Z$ & \multicolumn{2}{|l|}{8} \\
\hline Density (calculated) & \multicolumn{2}{|l|}{$1.235 \mathrm{Mg} / \mathrm{m}^{3}$} \\
\hline Absorption coefficient & \multicolumn{2}{|l|}{$0.079 \mathrm{~mm}^{-1}$} \\
\hline$F(000)$ & \multicolumn{2}{|l|}{1344} \\
\hline Crystal color, morphology & \multicolumn{2}{|l|}{ colorless, block } \\
\hline Crystal size & \multicolumn{2}{|c|}{$0.45 \times 0.32 \times 0.24 \mathrm{~mm}^{3}$} \\
\hline Theta range for data collection & \multicolumn{2}{|l|}{1.77 to $25.09^{\circ}$} \\
\hline Index ranges & \multicolumn{2}{|c|}{$-14 \square h \square 14,-27 \square k \square 27,-15 \square l \square 15$} \\
\hline Reflections collected & \multicolumn{2}{|l|}{33186} \\
\hline Independent reflections & \multicolumn{2}{|c|}{$6063[R($ int $)=0.0386]$} \\
\hline Observed reflections & \multicolumn{2}{|l|}{4523} \\
\hline Completeness to theta $=25.09^{\circ}$ & \multicolumn{2}{|l|}{$99.9 \%$} \\
\hline Absorption correction & \multicolumn{2}{|c|}{ Semi-empirical from equivalents } \\
\hline Max. and min. transmission & \multicolumn{2}{|c|}{1.000000 and 0.946704} \\
\hline Refinement method & \multicolumn{2}{|c|}{ Full-matrix least-squares on $F^{2}$} \\
\hline Data / restraints / parameters & \multicolumn{2}{|l|}{6063 / $31 / 482$} \\
\hline Goodness-of-fit on $F^{2}$ & \multicolumn{2}{|l|}{1.004} \\
\hline Final $R$ indices $[I>2 \operatorname{sigma}(I)]$ & \multicolumn{2}{|c|}{$R 1=0.0368, w R 2=0.0858$} \\
\hline$R$ indices (all data) & \multicolumn{2}{|c|}{$R 1=0.0569, w R 2=0.1004$} \\
\hline Largest diff. peak and hole & \multicolumn{2}{|c|}{0.229 and -0.198 e. $\AA^{-3}$} \\
\hline
\end{tabular}


Table 2. Atomic coordinates (x $\left.10^{4}\right)$ and equivalent isotropic displacement parameters $\left(\AA^{2} \times 10^{3}\right)$ for 03139. $U_{e q}$ is defined as one third of the trace of the orthogonalized $U_{i j}$ tensor.

\begin{tabular}{|c|c|c|c|c|}
\hline & $\mathrm{x}$ & $\mathrm{y}$ & $\mathrm{zU}_{\mathrm{eq}}$ & \\
\hline O1 & $3643(1)$ & $2258(1)$ & 1833(1) & $40(1)$ \\
\hline $\mathrm{O} 2$ & $3405(1)$ & $2936(1)$ & 3007(1) & $48(1)$ \\
\hline N1 & $3069(1)$ & $3003(1)$ & $-693(1)$ & $35(1)$ \\
\hline $\mathrm{C} 1$ & $3392(1)$ & $2822(1)$ & 2081(1) & $35(1)$ \\
\hline $\mathrm{C} 2$ & $3155(1)$ & $3165(1)$ & 1134(1) & $29(1)$ \\
\hline C3 & $3248(1)$ & 2821(1) & $306(1)$ & $29(1)$ \\
\hline $\mathrm{C} 4$ & $3540(1)$ & $2215(1)$ & $698(1)$ & $36(1)$ \\
\hline C5 & 2794(1) & $3760(1)$ & $925(1)$ & $29(1)$ \\
\hline C6 & $2554(1)$ & $3969(1)$ & $-69(1)$ & $31(1)$ \\
\hline C7 & 2729(1) & 3611(1) & $-1028(1)$ & $30(1)$ \\
\hline $\mathrm{C} 8$ & $3729(1)$ & $3874(1)$ & $-1420(1)$ & $37(1)$ \\
\hline C9 & 1593(1) & $3566(1)$ & $-1919(1)$ & $32(1)$ \\
\hline $\mathrm{C} 10$ & $1476(2)$ & 3773(1) & $-2945(1)$ & $46(1)$ \\
\hline C11 & $451(2)$ & $3689(1)$ & $-3732(2)$ & $64(1)$ \\
\hline $\mathrm{C} 12$ & $-454(2)$ & $3400(1)$ & $-3507(2)$ & $64(1)$ \\
\hline $\mathrm{C} 13$ & $-364(2)$ & $3197(1)$ & $-2492(2)$ & $58(1)$ \\
\hline $\mathrm{C} 14$ & $659(1)$ & $3280(1)$ & $-1695(1)$ & $44(1)$ \\
\hline $\mathrm{C} 15$ & $2078(2)$ & $4576(1)$ & $-348(1)$ & $47(1)$ \\
\hline C16 & $2240(1)$ & $4976(1)$ & $603(1)$ & $36(1)$ \\
\hline $\mathrm{C} 17$ & $1365(2)$ & $5056(1)$ & 1116(1) & $41(1)$ \\
\hline $\mathrm{C} 18$ & $1505(2)$ & $5438(1)$ & 1972(1) & $48(1)$ \\
\hline C19 & $2528(2)$ & $5738(1)$ & 2328(1) & $50(1)$ \\
\hline $\mathrm{C} 20$ & $3419(2)$ & $5653(1)$ & 1843(1) & $49(1)$ \\
\hline $\mathrm{C} 21$ & $3274(2)$ & $5274(1)$ & $990(1)$ & $44(1)$ \\
\hline $\mathrm{O} 3$ & $6189(1)$ & $2279(1)$ & 4955(1) & $44(1)$ \\
\hline $\mathrm{O} 4$ & $6482(1)$ & $2947(1)$ & $6261(1)$ & $45(1)$ \\
\hline N2 & $6558(1)$ & $3046(1)$ & 2644(1) & $40(1)$ \\
\hline $\mathrm{C} 22$ & $6443(1)$ & $2841(1)$ & 5323(1) & $36(1)$ \\
\hline $\mathrm{C} 23$ & $6605(1)$ & 3193(1) & 4470(1) & $32(1)$ \\
\hline $\mathrm{C} 24$ & $6472(1)$ & $2854(1)$ & $3584(1)$ & $32(1)$ \\
\hline $\mathrm{C} 25$ & $6212(2)$ & $2246(1)$ & 3848(1) & $40(1)$ \\
\hline
\end{tabular}




\begin{tabular}{|c|c|c|c|c|}
\hline $\mathrm{C} 26$ & $6868(2)$ & $3801(1)$ & $4400(1)$ & $40(1)$ \\
\hline $\mathrm{C} 28$ & $6917(1)$ & $3651(1)$ & $2476(1)$ & $36(1)$ \\
\hline $\mathrm{C} 29$ & $5964(2)$ & $3902(1)$ & $1556(2)$ & $53(1)$ \\
\hline $\mathrm{C} 30$ & $8093(1)$ & $3605(1)$ & $2210(1)$ & $38(1)$ \\
\hline $\mathrm{C} 31$ & $8342(15)$ & $3686(16)$ & $1237(12)$ & $49(2)$ \\
\hline C32 & $9482(9)$ & $3593(7)$ & $1173(8)$ & $62(2)$ \\
\hline $\mathrm{C} 33$ & $10336(7)$ & $3422(4)$ & $2046(7)$ & $65(2)$ \\
\hline $\mathrm{C} 34$ & 10083(8) & $3333(5)$ & $3019(6)$ & $64(2)$ \\
\hline C35 & $8973(10)$ & $3436(9)$ & $3113(8)$ & $49(2)$ \\
\hline C30' & 8093(1) & $3605(1)$ & $2210(1)$ & $38(1)$ \\
\hline C31' & $8139(15)$ & $3753(17)$ & $1170(12)$ & $49(2)$ \\
\hline C32' & $9149(9)$ & $3665(7)$ & $812(8)$ & $62(2)$ \\
\hline C33' & $10115(8)$ & $3421(5)$ & $1515(7)$ & $65(2)$ \\
\hline C34' & $10105(8)$ & $3291(5)$ & $2545(7)$ & $64(2)$ \\
\hline C35' & $9103(10)$ & $3371(10)$ & $2878(8)$ & $49(2)$ \\
\hline $\mathrm{C} 27$ & $6854(6)$ & 4038(3) & $3444(4)$ & $32(2)$ \\
\hline $\mathrm{C} 36$ & 7011(4) & $4682(2)$ & $3229(3)$ & $46(1)$ \\
\hline C37 & $7153(12)$ & $5060(13)$ & $4215(19)$ & $40(1)$ \\
\hline C38 & $8245(6)$ & $5127(3)$ & $4919(5)$ & $52(1)$ \\
\hline C39 & $8366(5)$ & $5496(2)$ & $5822(3)$ & $60(1)$ \\
\hline $\mathrm{C} 40$ & $7384(7)$ & $5786(2)$ & $5979(5)$ & $68(2)$ \\
\hline $\mathrm{C} 41$ & $6352(8)$ & $5697(3)$ & $5293(6)$ & $68(1)$ \\
\hline $\mathrm{C} 42$ & $6209(6)$ & $5338(2)$ & $4426(4)$ & $51(1)$ \\
\hline $\mathrm{C} 27^{\prime}$ & $7208(7)$ & 3992(3) & $3548(4)$ & $32(2)$ \\
\hline C36' & $7668(4)$ & $4605(2)$ & $3525(3)$ & $46(1)$ \\
\hline C37' & $7245(12)$ & $5030(13)$ & $4238(19)$ & $40(1)$ \\
\hline C38' & $7964(7)$ & $5215(3)$ & $5203(5)$ & $52(1)$ \\
\hline C39' & $7536(7)$ & $5608(2)$ & $5871(5)$ & $60(1)$ \\
\hline $\mathrm{C}^{\prime} 0^{\prime}$ & $6390(9)$ & 5793(3) & $5529(6)$ & $68(2)$ \\
\hline C41' & $5712(5)$ & $5622(2)$ & $4568(4)$ & $68(1)$ \\
\hline C42' & $6129(6)$ & $5240(3)$ & $3931(4)$ & $51(1)$ \\
\hline
\end{tabular}


Table 3. Bond lengths $[\AA]$ and angles $\left[^{\circ}\right]$ for 03139 .

\begin{tabular}{|c|c|c|c|}
\hline $\mathrm{O}(1)-\mathrm{C}(1)$ & $1.3862(18)$ & $\mathrm{C}(15)-\mathrm{H}(15 \mathrm{~B})$ & 0.9900 \\
\hline $\mathrm{O}(1)-\mathrm{C}(4)$ & $1.4394(18)$ & $C(16)-C(17)$ & $1.382(2)$ \\
\hline $\mathrm{O}(2)-\mathrm{C}(1)$ & $1.2189(18)$ & $C(16)-C(21)$ & $1.385(2)$ \\
\hline $\mathrm{N}(1)-\mathrm{C}(3)$ & $1.3186(18)$ & $C(17)-C(18)$ & $1.387(2)$ \\
\hline $\mathrm{N}(1)-\mathrm{C}(7)$ & $1.4883(18)$ & $\mathrm{C}(17)-\mathrm{H}(17 \mathrm{~A})$ & 0.9500 \\
\hline $\mathrm{N}(1)-\mathrm{H}(1 \mathrm{~N})$ & $0.918(17)$ & $\mathrm{C}(18)-\mathrm{C}(19)$ & $1.373(3)$ \\
\hline$C(1)-C(2)$ & $1.421(2)$ & $\mathrm{C}(18)-\mathrm{H}(18 \mathrm{~A})$ & 0.9500 \\
\hline$C(2)-C(3)$ & $1.355(2)$ & $C(19)-C(20)$ & $1.376(3)$ \\
\hline$C(2)-C(5)$ & $1.441(2)$ & $\mathrm{C}(19)-\mathrm{H}(19 \mathrm{~A})$ & 0.9500 \\
\hline$C(3)-C(4)$ & $1.494(2)$ & $C(20)-C(21)$ & $1.380(2)$ \\
\hline $\mathrm{C}(4)-\mathrm{H}(4 \mathrm{~A})$ & 0.9900 & $\mathrm{C}(20)-\mathrm{H}(20 \mathrm{~A})$ & 0.9500 \\
\hline $\mathrm{C}(4)-\mathrm{H}(4 \mathrm{~B})$ & 0.9900 & $\mathrm{C}(21)-\mathrm{H}(21 \mathrm{~A})$ & 0.9500 \\
\hline$C(5)-C(6)$ & $1.329(2)$ & $\mathrm{O}(3)-\mathrm{C}(22)$ & $1.3843(19)$ \\
\hline $\mathrm{C}(5)-\mathrm{H}(5 \mathrm{~A})$ & 0.9500 & $\mathrm{O}(3)-\mathrm{C}(25)$ & $1.4358(19)$ \\
\hline$C(6)-C(15)$ & $1.516(2)$ & $\mathrm{O}(4)-\mathrm{C}(22)$ & $1.2224(18)$ \\
\hline$C(6)-C(7)$ & $1.5424(19)$ & $\mathrm{N}(2)-\mathrm{C}(24)$ & $1.3184(19)$ \\
\hline$C(7)-C(8)$ & $1.532(2)$ & $\mathrm{N}(2)-\mathrm{C}(28)$ & $1.4888(19)$ \\
\hline$C(7)-C(9)$ & $1.537(2)$ & $\mathrm{N}(2)-\mathrm{H}(2 \mathrm{~N})$ & $0.910(18)$ \\
\hline $\mathrm{C}(8)-\mathrm{H}(8 \mathrm{~A})$ & 0.9800 & $C(22)-C(23)$ & $1.420(2)$ \\
\hline $\mathrm{C}(8)-\mathrm{H}(8 \mathrm{~B})$ & 0.9800 & $C(23)-C(24)$ & $1.358(2)$ \\
\hline $\mathrm{C}(8)-\mathrm{H}(8 \mathrm{C})$ & 0.9800 & $C(23)-C(26)$ & $1.442(2)$ \\
\hline $\mathrm{C}(9)-\mathrm{C}(10)$ & $1.379(2)$ & $C(24)-C(25)$ & $1.490(2)$ \\
\hline$C(9)-C(14)$ & $1.384(2)$ & $\mathrm{C}(25)-\mathrm{H}(25 \mathrm{~A})$ & 0.9900 \\
\hline$C(10)-C(11)$ & $1.386(2)$ & $\mathrm{C}(25)-\mathrm{H}(25 \mathrm{~B})$ & 0.9900 \\
\hline $\mathrm{C}(10)-\mathrm{H}(10 \mathrm{~A})$ & 0.9500 & $C(26)-C\left(27^{\prime}\right)$ & $1.339(5)$ \\
\hline$C(11)-C(12)$ & $1.361(3)$ & $C(26)-C(27)$ & $1.344(5)$ \\
\hline $\mathrm{C}(11)-\mathrm{H}(11 \mathrm{~A})$ & 0.9500 & $\mathrm{C}(26)-\mathrm{H}(26 \mathrm{~A})$ & 0.9500 \\
\hline$C(12)-C(13)$ & $1.368(3)$ & $C(28)-C(30)$ & $1.528(2)$ \\
\hline $\mathrm{C}(12)-\mathrm{H}(12 \mathrm{~A})$ & 0.9500 & $\mathrm{C}(28)-\mathrm{C}(29)$ & $1.529(2)$ \\
\hline$C(13)-C(14)$ & $1.391(2)$ & $\mathrm{C}(28)-\mathrm{C}\left(27^{\prime}\right)$ & $1.549(5)$ \\
\hline $\mathrm{C}(13)-\mathrm{H}(13 \mathrm{~A})$ & 0.9500 & $\mathrm{C}(28)-\mathrm{C}(27)$ & $1.549(5)$ \\
\hline $\mathrm{C}(14)-\mathrm{H}(14 \mathrm{~A})$ & 0.9500 & $\mathrm{C}(29)-\mathrm{H}(29 \mathrm{~A})$ & 0.9800 \\
\hline$C(15)-C(16)$ & $1.506(2)$ & $\mathrm{C}(29)-\mathrm{H}(29 \mathrm{~B})$ & 0.9800 \\
\hline $\mathrm{C}(15)-\mathrm{H}(15 \mathrm{~A})$ & 0.9900 & $\mathrm{C}(29)-\mathrm{H}(29 \mathrm{C})$ & 0.9800 \\
\hline
\end{tabular}




\begin{tabular}{|c|c|c|c|}
\hline$C(30)-C(31)$ & $1.372(9)$ & $\mathrm{C}\left(36^{\prime}\right)-\mathrm{C}\left(37^{\prime}\right)$ & $1.514(9)$ \\
\hline$C(30)-C(35)$ & $1.407(9)$ & $\mathrm{C}\left(36^{\prime}\right)-\mathrm{H}(36 \mathrm{C})$ & 0.9900 \\
\hline$C(31)-C(32)$ & $1.397(10)$ & $\mathrm{C}\left(36^{\prime}\right)-\mathrm{H}(36 \mathrm{D})$ & 0.9900 \\
\hline $\mathrm{C}(31)-\mathrm{H}(31 \mathrm{~A})$ & 0.9500 & $C\left(37^{\prime}\right)-C\left(42^{\prime}\right)$ & $1.373(10)$ \\
\hline$C(32)-C(33)$ & $1.367(8)$ & $\mathrm{C}\left(37^{\prime}\right)-\mathrm{C}\left(38^{\prime}\right)$ & $1.384(10)$ \\
\hline $\mathrm{C}(32)-\mathrm{H}(32 \mathrm{~A})$ & 0.9500 & $\mathrm{C}\left(38^{\prime}\right)-\mathrm{C}\left(39^{\prime}\right)$ & $1.430(7)$ \\
\hline$C(33)-C(34)$ & $1.378(8)$ & $\mathrm{C}\left(38^{\prime}\right)-\mathrm{H}\left(38^{\prime}\right)$ & 0.9500 \\
\hline $\mathrm{C}(33)-\mathrm{H}(33 \mathrm{~A})$ & 0.9500 & $\mathrm{C}\left(39^{\prime}\right)-\mathrm{C}\left(40^{\prime}\right)$ & $1.389(14)$ \\
\hline$C(34)-C(35)$ & $1.379(9)$ & $\mathrm{C}\left(39^{\prime}\right)-\mathrm{H}\left(39^{\prime}\right)$ & 0.9500 \\
\hline $\mathrm{C}(34)-\mathrm{H}(34 \mathrm{~A})$ & 0.9500 & $\mathrm{C}\left(40^{\prime}\right)-\mathrm{C}\left(41^{\prime}\right)$ & $1.352(10)$ \\
\hline $\mathrm{C}(35)-\mathrm{H}(35 \mathrm{~A})$ & 0.9500 & $\mathrm{C}\left(40^{\prime}\right)-\mathrm{H}\left(40^{\prime}\right)$ & 0.9500 \\
\hline $\mathrm{C}\left(31^{\prime}\right)-\mathrm{C}\left(32^{\prime}\right)$ & $1.409(10)$ & $\mathrm{C}\left(41^{\prime}\right)-\mathrm{C}\left(42^{\prime}\right)$ & $1.380(6)$ \\
\hline $\mathrm{C}\left(31^{\prime}\right)-\mathrm{H}(31 \mathrm{~B})$ & 0.9500 & $\mathrm{C}\left(41^{\prime}\right)-\mathrm{H}\left(41^{\prime}\right)$ & 0.9500 \\
\hline $\mathrm{C}\left(32^{\prime}\right)-\mathrm{C}\left(33^{\prime}\right)$ & $1.386(8)$ & $\mathrm{C}\left(42^{\prime}\right)-\mathrm{H}\left(42^{\prime}\right)$ & 0.9500 \\
\hline $\mathrm{C}\left(32^{\prime}\right)-\mathrm{H}(32 \mathrm{~B})$ & 0.9500 & $\mathrm{C}(1)-\mathrm{O}(1)-\mathrm{C}(4)$ & $109.35(11)$ \\
\hline C(33')-C(34') & $1.365(9)$ & $\mathrm{C}(3)-\mathrm{N}(1)-\mathrm{C}(7)$ & $123.08(12)$ \\
\hline $\mathrm{C}\left(33^{\prime}\right)-\mathrm{H}(33 \mathrm{~B})$ & 0.9500 & $\mathrm{C}(3)-\mathrm{N}(1)-\mathrm{H}(1 \mathrm{~N})$ & $118.5(10)$ \\
\hline $\mathrm{C}\left(34^{\prime}\right)-\mathrm{C}\left(35^{\prime}\right)$ & $1.379(9)$ & $\mathrm{C}(7)-\mathrm{N}(1)-\mathrm{H}(1 \mathrm{~N})$ & $118.4(10)$ \\
\hline $\mathrm{C}\left(34^{\prime}\right)-\mathrm{H}(34 \mathrm{~B})$ & 0.9500 & $\mathrm{O}(2)-\mathrm{C}(1)-\mathrm{O}(1)$ & 118.61(13) \\
\hline $\mathrm{C}\left(35^{\prime}\right)-\mathrm{H}(35 \mathrm{~B})$ & 0.9500 & $\mathrm{O}(2)-\mathrm{C}(1)-\mathrm{C}(2)$ & $132.18(15)$ \\
\hline$C(27)-C(36)$ & $1.529(7)$ & $\mathrm{O}(1)-\mathrm{C}(1)-\mathrm{C}(2)$ & $109.21(13)$ \\
\hline$C(36)-C(37)$ & $1.513(9)$ & $\mathrm{C}(3)-\mathrm{C}(2)-\mathrm{C}(1)$ & $108.30(13)$ \\
\hline $\mathrm{C}(36)-\mathrm{H}(36 \mathrm{~A})$ & 0.9900 & $C(3)-C(2)-C(5)$ & $119.28(13)$ \\
\hline $\mathrm{C}(36)-\mathrm{H}(36 \mathrm{~B})$ & 0.9900 & $C(1)-C(2)-C(5)$ & $132.30(13)$ \\
\hline$C(37)-C(42)$ & $1.379(10)$ & $\mathrm{N}(1)-\mathrm{C}(3)-\mathrm{C}(2)$ & $123.90(13)$ \\
\hline$C(37)-C(38)$ & $1.391(10)$ & $\mathrm{N}(1)-\mathrm{C}(3)-\mathrm{C}(4)$ & $126.38(13)$ \\
\hline C(38)-C(39) & $1.417(6)$ & $\mathrm{C}(2)-\mathrm{C}(3)-\mathrm{C}(4)$ & 109.69(12) \\
\hline $\mathrm{C}(38)-\mathrm{H}(38)$ & 0.9500 & $\mathrm{O}(1)-\mathrm{C}(4)-\mathrm{C}(3)$ & $103.39(11)$ \\
\hline$C(39)-C(40)$ & $1.406(9)$ & $\mathrm{O}(1)-\mathrm{C}(4)-\mathrm{H}(4 \mathrm{~A})$ & 111.1 \\
\hline $\mathrm{C}(39)-\mathrm{H}(39)$ & 0.9500 & $\mathrm{C}(3)-\mathrm{C}(4)-\mathrm{H}(4 \mathrm{~A})$ & 111.1 \\
\hline $\mathrm{C}(40)-\mathrm{C}(41)$ & $1.333(14)$ & $\mathrm{O}(1)-\mathrm{C}(4)-\mathrm{H}(4 \mathrm{~B})$ & 111.1 \\
\hline $\mathrm{C}(40)-\mathrm{H}(40)$ & 0.9500 & $\mathrm{C}(3)-\mathrm{C}(4)-\mathrm{H}(4 \mathrm{~B})$ & 111.1 \\
\hline$C(41)-C(42)$ & $1.365(7)$ & $\mathrm{H}(4 \mathrm{~A})-\mathrm{C}(4)-\mathrm{H}(4 \mathrm{~B})$ & 109.0 \\
\hline $\mathrm{C}(41)-\mathrm{H}(41)$ & 0.9500 & $C(6)-C(5)-C(2)$ & $120.15(13)$ \\
\hline $\mathrm{C}(42)-\mathrm{H}(42)$ & 0.9500 & $\mathrm{C}(6)-\mathrm{C}(5)-\mathrm{H}(5 \mathrm{~A})$ & 119.9 \\
\hline $\mathrm{C}\left(27^{\prime}\right)-\mathrm{C}\left(36^{\prime}\right)$ & $1.517(7)$ & $\mathrm{C}(2)-\mathrm{C}(5)-\mathrm{H}(5 \mathrm{~A})$ & 119.9 \\
\hline
\end{tabular}




\begin{tabular}{|c|c|c|c|}
\hline$C(5)-C(6)-C(15)$ & $122.37(13)$ & $\mathrm{C}(16)-\mathrm{C}(15)-\mathrm{H}(15 \mathrm{~B})$ & 108.7 \\
\hline$C(5)-C(6)-C(7)$ & $122.73(13)$ & $\mathrm{C}(6)-\mathrm{C}(15)-\mathrm{H}(15 \mathrm{~B})$ & 108.7 \\
\hline$C(15)-C(6)-C(7)$ & $114.90(12)$ & $\mathrm{H}(15 \mathrm{~A})-\mathrm{C}(15)-\mathrm{H}(15 \mathrm{~B})$ & 107.6 \\
\hline $\mathrm{N}(1)-\mathrm{C}(7)-\mathrm{C}(8)$ & $106.86(12)$ & $\mathrm{C}(17)-\mathrm{C}(16)-\mathrm{C}(21)$ & $118.17(15)$ \\
\hline $\mathrm{N}(1)-\mathrm{C}(7)-\mathrm{C}(9)$ & 105.93(11) & $\mathrm{C}(17)-\mathrm{C}(16)-\mathrm{C}(15)$ & $121.02(15)$ \\
\hline C(8)-C(7)-C(9) & $112.95(12)$ & $C(21)-C(16)-C(15)$ & $120.81(15)$ \\
\hline $\mathrm{N}(1)-\mathrm{C}(7)-\mathrm{C}(6)$ & $110.59(11)$ & $C(16)-C(17)-C(18)$ & $120.82(16)$ \\
\hline$C(8)-C(7)-C(6)$ & $109.23(12)$ & $\mathrm{C}(16)-\mathrm{C}(17)-\mathrm{H}(17 \mathrm{~A})$ & 119.6 \\
\hline $\mathrm{C}(9)-\mathrm{C}(7)-\mathrm{C}(6)$ & $111.16(11)$ & $\mathrm{C}(18)-\mathrm{C}(17)-\mathrm{H}(17 \mathrm{~A})$ & 119.6 \\
\hline $\mathrm{C}(7)-\mathrm{C}(8)-\mathrm{H}(8 \mathrm{~A})$ & 109.5 & $\mathrm{C}(19)-\mathrm{C}(18)-\mathrm{C}(17)$ & $120.09(17)$ \\
\hline $\mathrm{C}(7)-\mathrm{C}(8)-\mathrm{H}(8 \mathrm{~B})$ & 109.5 & $\mathrm{C}(19)-\mathrm{C}(18)-\mathrm{H}(18 \mathrm{~A})$ & 120.0 \\
\hline $\mathrm{H}(8 \mathrm{~A})-\mathrm{C}(8)-\mathrm{H}(8 \mathrm{~B})$ & 109.5 & $\mathrm{C}(17)-\mathrm{C}(18)-\mathrm{H}(18 \mathrm{~A})$ & 120.0 \\
\hline $\mathrm{C}(7)-\mathrm{C}(8)-\mathrm{H}(8 \mathrm{C})$ & 109.5 & $C(18)-C(19)-C(20)$ & $119.82(16)$ \\
\hline $\mathrm{H}(8 \mathrm{~A})-\mathrm{C}(8)-\mathrm{H}(8 \mathrm{C})$ & 109.5 & $\mathrm{C}(18)-\mathrm{C}(19)-\mathrm{H}(19 \mathrm{~A})$ & 120.1 \\
\hline $\mathrm{H}(8 \mathrm{~B})-\mathrm{C}(8)-\mathrm{H}(8 \mathrm{C})$ & 109.5 & $\mathrm{C}(20)-\mathrm{C}(19)-\mathrm{H}(19 \mathrm{~A})$ & 120.1 \\
\hline C(10)-C(9)-C(14) & $118.36(15)$ & $C(19)-C(20)-C(21)$ & $119.90(17)$ \\
\hline C(10)-C(9)-C(7) & $123.11(14)$ & $\mathrm{C}(19)-\mathrm{C}(20)-\mathrm{H}(20 \mathrm{~A})$ & 120.0 \\
\hline $\mathrm{C}(14)-\mathrm{C}(9)-\mathrm{C}(7)$ & $118.47(13)$ & $\mathrm{C}(21)-\mathrm{C}(20)-\mathrm{H}(20 \mathrm{~A})$ & 120.0 \\
\hline C(9)-C(10)-C(11) & $120.49(17)$ & $\mathrm{C}(20)-\mathrm{C}(21)-\mathrm{C}(16)$ & $121.16(16)$ \\
\hline $\mathrm{C}(9)-\mathrm{C}(10)-\mathrm{H}(10 \mathrm{~A})$ & 119.8 & $\mathrm{C}(20)-\mathrm{C}(21)-\mathrm{H}(21 \mathrm{~A})$ & 119.4 \\
\hline $\mathrm{C}(11)-\mathrm{C}(10)-\mathrm{H}(10 \mathrm{~A})$ & 119.8 & $\mathrm{C}(16)-\mathrm{C}(21)-\mathrm{H}(21 \mathrm{~A})$ & 119.4 \\
\hline $\mathrm{C}(12)-\mathrm{C}(11)-\mathrm{C}(10)$ & $120.63(19)$ & $\mathrm{C}(22)-\mathrm{O}(3)-\mathrm{C}(25)$ & $109.34(11)$ \\
\hline $\mathrm{C}(12)-\mathrm{C}(11)-\mathrm{H}(11 \mathrm{~A})$ & 119.7 & $\mathrm{C}(24)-\mathrm{N}(2)-\mathrm{C}(28)$ & $122.69(13)$ \\
\hline $\mathrm{C}(10)-\mathrm{C}(11)-\mathrm{H}(11 \mathrm{~A})$ & 119.7 & $\mathrm{C}(24)-\mathrm{N}(2)-\mathrm{H}(2 \mathrm{~N})$ & 118.6(11) \\
\hline $\mathrm{C}(11)-\mathrm{C}(12)-\mathrm{C}(13)$ & $119.92(18)$ & $\mathrm{C}(28)-\mathrm{N}(2)-\mathrm{H}(2 \mathrm{~N})$ & $118.2(11)$ \\
\hline $\mathrm{C}(11)-\mathrm{C}(12)-\mathrm{H}(12 \mathrm{~A})$ & 120.0 & $\mathrm{O}(4)-\mathrm{C}(22)-\mathrm{O}(3)$ & $118.48(14)$ \\
\hline $\mathrm{C}(13)-\mathrm{C}(12)-\mathrm{H}(12 \mathrm{~A})$ & 120.0 & $\mathrm{O}(4)-\mathrm{C}(22)-\mathrm{C}(23)$ & $132.31(15)$ \\
\hline$C(12)-C(13)-C(14)$ & $119.90(18)$ & $\mathrm{O}(3)-\mathrm{C}(22)-\mathrm{C}(23)$ & $109.21(13)$ \\
\hline $\mathrm{C}(12)-\mathrm{C}(13)-\mathrm{H}(13 \mathrm{~A})$ & 120.0 & $C(24)-C(23)-C(22)$ & $108.24(13)$ \\
\hline $\mathrm{C}(14)-\mathrm{C}(13)-\mathrm{H}(13 \mathrm{~A})$ & 120.0 & $\mathrm{C}(24)-\mathrm{C}(23)-\mathrm{C}(26)$ & $119.09(14)$ \\
\hline C(9)-C(14)-C(13) & $120.68(17)$ & $C(22)-C(23)-C(26)$ & $132.67(14)$ \\
\hline $\mathrm{C}(9)-\mathrm{C}(14)-\mathrm{H}(14 \mathrm{~A})$ & 119.7 & N(2)-C(24)-C(23) & $124.06(14)$ \\
\hline $\mathrm{C}(13)-\mathrm{C}(14)-\mathrm{H}(14 \mathrm{~A})$ & 119.7 & $\mathrm{~N}(2)-\mathrm{C}(24)-\mathrm{C}(25)$ & $126.41(14)$ \\
\hline$C(16)-C(15)-C(6)$ & $114.15(12)$ & $C(23)-C(24)-C(25)$ & $109.53(13)$ \\
\hline $\mathrm{C}(16)-\mathrm{C}(15)-\mathrm{H}(15 \mathrm{~A})$ & 108.7 & $\mathrm{O}(3)-\mathrm{C}(25)-\mathrm{C}(24)$ & $103.64(12)$ \\
\hline $\mathrm{C}(6)-\mathrm{C}(15)-\mathrm{H}(15 \mathrm{~A})$ & 108.7 & $\mathrm{O}(3)-\mathrm{C}(25)-\mathrm{H}(25 \mathrm{~A})$ & 111.0 \\
\hline
\end{tabular}




\begin{tabular}{|c|c|}
\hline $\mathrm{C}(24)-\mathrm{C}(25)-\mathrm{H}(25 \mathrm{~A})$ & 111.0 \\
\hline $\mathrm{O}(3)-\mathrm{C}(25)-\mathrm{H}(25 \mathrm{~B})$ & 111.0 \\
\hline $\mathrm{C}(24)-\mathrm{C}(25)-\mathrm{H}(25 \mathrm{~B})$ & 111.0 \\
\hline $\mathrm{H}(25 \mathrm{~A})-\mathrm{C}(25)-\mathrm{H}(25 \mathrm{~B})$ & 109.0 \\
\hline$C\left(27^{\prime}\right)-C(26)-C(27)$ & 18.1(6) \\
\hline$C\left(27^{\prime}\right)-C(26)-C(23)$ & $119.0(3)$ \\
\hline $\mathrm{C}(27)-\mathrm{C}(26)-\mathrm{C}(23)$ & $119.9(3)$ \\
\hline $\mathrm{C}\left(27^{\prime}\right)-\mathrm{C}(26)-\mathrm{H}(26 \mathrm{~A})$ & 117.7 \\
\hline $\mathrm{C}(27)-\mathrm{C}(26)-\mathrm{H}(26 \mathrm{~A})$ & 120.1 \\
\hline$C(23)-C(26)-H(26 A)$ & 120.1 \\
\hline $\mathrm{N}(2)-\mathrm{C}(28)-\mathrm{C}(30)$ & $106.31(12)$ \\
\hline $\mathrm{N}(2)-\mathrm{C}(28)-\mathrm{C}(29)$ & $106.39(13)$ \\
\hline $\mathrm{C}(30)-\mathrm{C}(28)-\mathrm{C}(29)$ & $113.13(13)$ \\
\hline $\mathrm{N}(2)-\mathrm{C}(28)-\mathrm{C}\left(27^{\prime}\right)$ & $110.5(2)$ \\
\hline $\mathrm{C}(30)-\mathrm{C}(28)-\mathrm{C}\left(27^{\prime}\right)$ & $103.1(3)$ \\
\hline $\mathrm{C}(29)-\mathrm{C}(28)-\mathrm{C}\left(27^{\prime}\right)$ & $116.9(3)$ \\
\hline $\mathrm{N}(2)-\mathrm{C}(28)-\mathrm{C}(27)$ & $110.2(2)$ \\
\hline $\mathrm{C}(30)-\mathrm{C}(28)-\mathrm{C}(27)$ & $117.2(3)$ \\
\hline C(29)-C(28)-C(27) & $103.2(3)$ \\
\hline $\mathrm{C}\left(27^{\prime}\right)-\mathrm{C}(28)-\mathrm{C}(27)$ & $15.7(5)$ \\
\hline C(28)-C(29)-H(29A) & 109.5 \\
\hline C(28)-C(29)-H(29B) & 109.5 \\
\hline H(29A)-C(29)-H(29B) & 109.5 \\
\hline C(28)-C(29)-H(29C) & 109.5 \\
\hline H(29A)-C(29)-H(29C) & 109.5 \\
\hline H(29B)-C(29)-H(29C) & 109.5 \\
\hline $\mathrm{C}(31)-\mathrm{C}(30)-\mathrm{C}(35)$ & $120.3(7)$ \\
\hline $\mathrm{C}(31)-\mathrm{C}(30)-\mathrm{C}(28)$ & $128.3(6)$ \\
\hline $\mathrm{C}(35)-\mathrm{C}(30)-\mathrm{C}(28)$ & $111.3(4)$ \\
\hline $\mathrm{C}(30)-\mathrm{C}(31)-\mathrm{C}(32)$ & $118.3(10)$ \\
\hline $\mathrm{C}(30)-\mathrm{C}(31)-\mathrm{H}(31 \mathrm{~A})$ & 120.9 \\
\hline $\mathrm{C}(32)-\mathrm{C}(31)-\mathrm{H}(31 \mathrm{~A})$ & 120.9 \\
\hline C(33)-C(32)-C(31) & $121.7(9)$ \\
\hline $\mathrm{C}(33)-\mathrm{C}(32)-\mathrm{H}(32 \mathrm{~A})$ & 119.2 \\
\hline $\mathrm{C}(31)-\mathrm{C}(32)-\mathrm{H}(32 \mathrm{~A})$ & 119.2 \\
\hline C(32)-C(33)-C(34) & $120.1(8)$ \\
\hline
\end{tabular}

\begin{tabular}{|c|c|}
\hline $\mathrm{C}(32)-\mathrm{C}(33)-\mathrm{H}(33 \mathrm{~A})$ & 120.0 \\
\hline $\mathrm{C}(34)-\mathrm{C}(33)-\mathrm{H}(33 \mathrm{~A})$ & 120.0 \\
\hline $\mathrm{C}(33)-\mathrm{C}(34)-\mathrm{C}(35)$ & $119.6(8)$ \\
\hline $\mathrm{C}(33)-\mathrm{C}(34)-\mathrm{H}(34 \mathrm{~A})$ & 120.2 \\
\hline $\mathrm{C}(35)-\mathrm{C}(34)-\mathrm{H}(34 \mathrm{~A})$ & 120.2 \\
\hline$C(34)-C(35)-C(30)$ & $120.1(8)$ \\
\hline $\mathrm{C}(34)-\mathrm{C}(35)-\mathrm{H}(35 \mathrm{~A})$ & 120.0 \\
\hline $\mathrm{C}(30)-\mathrm{C}(35)-\mathrm{H}(35 \mathrm{~A})$ & 120.0 \\
\hline $\mathrm{C}\left(32^{\prime}\right)-\mathrm{C}\left(31^{\prime}\right)-\mathrm{H}(31 \mathrm{~B})$ & 119.2 \\
\hline $\mathrm{C}\left(33^{\prime}\right)-\mathrm{C}\left(32^{\prime}\right)-\mathrm{C}\left(31^{\prime}\right)$ & $118.7(9)$ \\
\hline $\mathrm{C}\left(33^{\prime}\right)-\mathrm{C}\left(32^{\prime}\right)-\mathrm{H}(32 \mathrm{~B})$ & 120.7 \\
\hline $\mathrm{C}\left(31^{\prime}\right)-\mathrm{C}\left(32^{\prime}\right)-\mathrm{H}(32 \mathrm{~B})$ & 120.7 \\
\hline $\mathrm{C}\left(34^{\prime}\right)-\mathrm{C}\left(33^{\prime}\right)-\mathrm{C}\left(32^{\prime}\right)$ & $120.8(8)$ \\
\hline $\mathrm{C}\left(34^{\prime}\right)-\mathrm{C}\left(33^{\prime}\right)-\mathrm{H}(33 \mathrm{~B})$ & 119.6 \\
\hline $\mathrm{C}\left(32^{\prime}\right)-\mathrm{C}\left(33^{\prime}\right)-\mathrm{H}(33 \mathrm{~B})$ & 119.6 \\
\hline $\mathrm{C}\left(33^{\prime}\right)-\mathrm{C}\left(34^{\prime}\right)-\mathrm{C}\left(35^{\prime}\right)$ & $119.8(8)$ \\
\hline $\mathrm{C}\left(33^{\prime}\right)-\mathrm{C}\left(34^{\prime}\right)-\mathrm{H}(34 \mathrm{~B})$ & 120.1 \\
\hline $\mathrm{C}\left(35^{\prime}\right)-\mathrm{C}\left(34^{\prime}\right)-\mathrm{H}(34 \mathrm{~B})$ & 120.1 \\
\hline $\mathrm{C}\left(34^{\prime}\right)-\mathrm{C}\left(35^{\prime}\right)-\mathrm{H}(35 \mathrm{~B})$ & 118.8 \\
\hline$C(26)-C(27)-C(36)$ & $125.9(4)$ \\
\hline $\mathrm{C}(26)-\mathrm{C}(27)-\mathrm{C}(28)$ & $120.9(4)$ \\
\hline $\mathrm{C}(36)-\mathrm{C}(27)-\mathrm{C}(28)$ & $112.2(3)$ \\
\hline$C(37)-C(36)-C(27)$ & $113.4(15)$ \\
\hline $\mathrm{C}(37)-\mathrm{C}(36)-\mathrm{H}(36 \mathrm{~A})$ & 108.9 \\
\hline $\mathrm{C}(27)-\mathrm{C}(36)-\mathrm{H}(36 \mathrm{~A})$ & 108.9 \\
\hline $\mathrm{C}(37)-\mathrm{C}(36)-\mathrm{H}(36 \mathrm{~B})$ & 108.9 \\
\hline $\mathrm{C}(27)-\mathrm{C}(36)-\mathrm{H}(36 \mathrm{~B})$ & 108.9 \\
\hline $\mathrm{H}(36 \mathrm{~A})-\mathrm{C}(36)-\mathrm{H}(36 \mathrm{~B})$ & 107.7 \\
\hline C(42)-C(37)-C(38) & $119.5(7)$ \\
\hline$C(42)-C(37)-C(36)$ & $120.7(7)$ \\
\hline C(38)-C(37)-C(36) & $119.8(7)$ \\
\hline C(37)-C(38)-C(39) & $118.9(6)$ \\
\hline $\mathrm{C}(37)-\mathrm{C}(38)-\mathrm{H}(38)$ & 120.5 \\
\hline C(39)-C(38)-H(38) & 120.5 \\
\hline C(40)-C(39)-C(38) & $119.3(5)$ \\
\hline C(40)-C(39)-H(39) & 120.4 \\
\hline
\end{tabular}




$\begin{array}{llll}\mathrm{C}(38)-\mathrm{C}(39)-\mathrm{H}(39) & 120.4 & \mathrm{C}\left(42^{\prime}\right)-\mathrm{C}\left(37^{\prime}\right)-\mathrm{C}\left(38^{\prime}\right) & 118.8(7) \\ \mathrm{C}(41)-\mathrm{C}(40)-\mathrm{C}(39) & 119.4(5) & \mathrm{C}\left(42^{\prime}\right)-\mathrm{C}\left(37^{\prime}\right)-\mathrm{C}\left(36^{\prime}\right) & 120.1(7) \\ \mathrm{C}(41)-\mathrm{C}(40)-\mathrm{H}(40) & 120.3 & \mathrm{C}\left(38^{\prime}\right)-\mathrm{C}\left(37^{\prime}\right)-\mathrm{C}\left(36^{\prime}\right) & 121.2(7) \\ \mathrm{C}(39)-\mathrm{C}(40)-\mathrm{H}(40) & 120.3 & \mathrm{C}\left(37^{\prime}\right)-\mathrm{C}\left(38^{\prime}\right)-\mathrm{C}\left(39^{\prime}\right) & 120.1(7) \\ \mathrm{C}(40)-\mathrm{C}(41)-\mathrm{C}(42) & 122.4(8) & \mathrm{C}\left(37^{\prime}\right)-\mathrm{C}\left(38^{\prime}\right)-\mathrm{H}\left(38^{\prime}\right) & 120.0 \\ \mathrm{C}(40)-\mathrm{C}(41)-\mathrm{H}(41) & 118.8 & \mathrm{C}\left(39^{\prime}\right)-\mathrm{C}\left(38^{\prime}\right)-\mathrm{H}\left(38^{\prime}\right) & 120.0 \\ \mathrm{C}(42)-\mathrm{C}(41)-\mathrm{H}(41) & 118.8 & \mathrm{C}\left(40^{\prime}\right)-\mathrm{C}\left(39^{\prime}\right)-\mathrm{C}\left(38^{\prime}\right) & 118.4(6) \\ \mathrm{C}(41)-\mathrm{C}(42)-\mathrm{C}(37) & 120.4(7) & \mathrm{C}\left(40^{\prime}\right)-\mathrm{C}\left(39^{\prime}\right)-\mathrm{H}\left(39^{\prime}\right) & 120.8 \\ \mathrm{C}(41)-\mathrm{C}(42)-\mathrm{H}(42) & 119.8 & \mathrm{C}\left(38^{\prime}\right)-\mathrm{C}\left(39^{\prime}\right)-\mathrm{H}\left(39^{\prime}\right) & 120.8 \\ \mathrm{C}(37)-\mathrm{C}(42)-\mathrm{H}(42) & 119.8 & \mathrm{C}\left(41^{\prime}\right)-\mathrm{C}\left(40^{\prime}\right)-\mathrm{C}\left(39^{\prime}\right) & 120.7(6) \\ \mathrm{C}(26)-\mathrm{C}\left(27^{\prime}\right)-\mathrm{C}\left(36^{\prime}\right) & 120.5(4) & \mathrm{C}\left(41^{\prime}\right)-\mathrm{C}\left(40^{\prime}\right)-\mathrm{H}\left(40^{\prime}\right) & 119.7 \\ \mathrm{C}(26)-\mathrm{C}\left(27^{\prime}\right)-\mathrm{C}(28) & 121.4(5) & \mathrm{C}\left(39^{\prime}\right)-\mathrm{C}\left(40^{\prime}\right)-\mathrm{H}\left(40^{\prime}\right) & 119.7 \\ \mathrm{C}\left(36^{\prime}\right)-\mathrm{C}\left(27^{\prime}\right)-\mathrm{C}(28) & 117.0(4) & \mathrm{C}\left(40^{\prime}\right)-\mathrm{C}\left(41^{\prime}\right)-\mathrm{C}\left(42^{\prime}\right) & 120.5(6) \\ \mathrm{C}\left(37^{\prime}\right)-\mathrm{C}\left(36^{\prime}\right)-\mathrm{C}\left(27^{\prime}\right) & 114.3(15) & \mathrm{C}\left(40^{\prime}\right)-\mathrm{C}\left(41^{\prime}\right)-\mathrm{H}\left(41^{\prime}\right) & 119.7 \\ \mathrm{C}\left(37^{\prime}\right)-\mathrm{C}\left(36^{\prime}\right)-\mathrm{H}(36 \mathrm{C}) & 108.7 & \mathrm{C}\left(42^{\prime}\right)-\mathrm{C}\left(41^{\prime}\right)-\mathrm{H}\left(41^{\prime}\right) & 119.7 \\ \mathrm{C}\left(27^{\prime}\right)-\mathrm{C}\left(36^{\prime}\right)-\mathrm{H}(36 \mathrm{C}) & 108.7 & \mathrm{C}\left(37^{\prime}\right)-\mathrm{C}\left(42^{\prime}\right)-\mathrm{C}\left(41^{\prime}\right) & 121.5(6) \\ \mathrm{C}\left(37^{\prime}\right)-\mathrm{C}\left(36^{\prime}\right)-\mathrm{H}(36 \mathrm{D}) & 108.7 & \mathrm{C}\left(37^{\prime}\right)-\mathrm{C}\left(42^{\prime}\right)-\mathrm{H}\left(42^{\prime}\right) & 119.3 \\ \mathrm{C}\left(27^{\prime}\right)-\mathrm{C}\left(36^{\prime}\right)-\mathrm{H}(36 \mathrm{D}) & 108.7 & \mathrm{C}\left(41^{\prime}\right)-\mathrm{C}\left(42^{\prime}\right)-\mathrm{H}\left(42^{\prime}\right) & 119.3 \\ \mathrm{H}(36 \mathrm{C})-\mathrm{C}\left(36^{\prime}\right)-\mathrm{H}(36 \mathrm{D}) & 107.6 & & \\ & & & \end{array}$

Symmetry transformations used to generate equivalent atoms: 
Table 4. Anisotropic displacement parameters $\left(\AA^{2} \times 10^{3}\right)$ for 03139. The anisotropic displacement factor exponent takes the form: $-2 \square^{2}\left[h^{2} a^{* 2} U_{11}+\ldots+2 h k a^{*} b^{*} U_{12}\right]$

\begin{tabular}{|c|c|c|c|c|c|c|}
\hline & $\mathrm{U}_{11}$ & $\mathrm{U}_{22}$ & $\mathrm{U}_{33}$ & $\mathrm{U}_{23}$ & $\mathrm{U}_{13}$ & $\mathrm{U}_{12}$ \\
\hline $\mathrm{O} 1$ & $56(1)$ & $29(1)$ & $36(1)$ & $6(1)$ & $11(1)$ & $2(1)$ \\
\hline $\mathrm{O} 2$ & $76(1)$ & $40(1)$ & $30(1)$ & $6(1)$ & $19(1)$ & $-1(1)$ \\
\hline N1 & $47(1)$ & $30(1)$ & $27(1)$ & $-2(1)$ & $9(1)$ & $9(1)$ \\
\hline $\mathrm{C} 1$ & $42(1)$ & $31(1)$ & $34(1)$ & $4(1)$ & $11(1)$ & $-3(1)$ \\
\hline $\mathrm{C} 2$ & $32(1)$ & $29(1)$ & $27(1)$ & $1(1)$ & $9(1)$ & $-1(1)$ \\
\hline $\mathrm{C} 3$ & $26(1)$ & $29(1)$ & $31(1)$ & $1(1)$ & $7(1)$ & $0(1)$ \\
\hline $\mathrm{C} 4$ & $41(1)$ & $29(1)$ & $36(1)$ & $1(1)$ & $9(1)$ & $1(1)$ \\
\hline $\mathrm{C} 5$ & $31(1)$ & $30(1)$ & $27(1)$ & $-3(1)$ & $8(1)$ & $1(1)$ \\
\hline C6 & $33(1)$ & $29(1)$ & $28(1)$ & $-2(1)$ & $6(1)$ & $3(1)$ \\
\hline $\mathrm{C} 7$ & $35(1)$ & $28(1)$ & $26(1)$ & $0(1)$ & $6(1)$ & $4(1)$ \\
\hline $\mathrm{C} 8$ & $36(1)$ & $39(1)$ & $37(1)$ & $-3(1)$ & $9(1)$ & $0(1)$ \\
\hline C9 & $34(1)$ & $28(1)$ & $31(1)$ & $-3(1)$ & $6(1)$ & $4(1)$ \\
\hline $\mathrm{C} 10$ & $46(1)$ & $57(1)$ & $33(1)$ & $5(1)$ & $4(1)$ & $1(1)$ \\
\hline $\mathrm{C} 11$ & $59(1)$ & $88(2)$ & $36(1)$ & $0(1)$ & $-4(1)$ & $7(1)$ \\
\hline $\mathrm{C} 12$ & $49(1)$ & $73(1)$ & $56(1)$ & $-18(1)$ & $-12(1)$ & $8(1)$ \\
\hline $\mathrm{C} 13$ & $35(1)$ & $50(1)$ & $85(2)$ & $-10(1)$ & $9(1)$ & $-5(1)$ \\
\hline $\mathrm{C} 14$ & $41(1)$ & $43(1)$ & $49(1)$ & $2(1)$ & $12(1)$ & $1(1)$ \\
\hline $\mathrm{C} 15$ & $71(1)$ & $38(1)$ & $29(1)$ & $0(1)$ & $7(1)$ & $19(1)$ \\
\hline C16 & $51(1)$ & $27(1)$ & $29(1)$ & $3(1)$ & $7(1)$ & 11(1) \\
\hline $\mathrm{C} 17$ & $43(1)$ & $38(1)$ & $40(1)$ & $-3(1)$ & $5(1)$ & $5(1)$ \\
\hline $\mathrm{C} 18$ & $53(1)$ & $49(1)$ & $43(1)$ & $-6(1)$ & $16(1)$ & $10(1)$ \\
\hline C19 & $67(1)$ & $40(1)$ & $39(1)$ & $-9(1)$ & $5(1)$ & $3(1)$ \\
\hline $\mathrm{C} 20$ & $53(1)$ & $40(1)$ & $50(1)$ & $2(1)$ & $4(1)$ & $-7(1)$ \\
\hline $\mathrm{C} 21$ & $52(1)$ & $41(1)$ & $44(1)$ & $9(1)$ & $17(1)$ & $6(1)$ \\
\hline $\mathrm{O} 3$ & $55(1)$ & $33(1)$ & $42(1)$ & $6(1)$ & $9(1)$ & $-7(1)$ \\
\hline $\mathrm{O} 4$ & $57(1)$ & $47(1)$ & $33(1)$ & $6(1)$ & $12(1)$ & $-6(1)$ \\
\hline $\mathrm{N} 2$ & $54(1)$ & $34(1)$ & $37(1)$ & $-9(1)$ & $20(1)$ & $-11(1)$ \\
\hline $\mathrm{C} 22$ & $33(1)$ & $36(1)$ & $38(1)$ & $4(1)$ & $6(1)$ & $-2(1)$ \\
\hline $\mathrm{C} 23$ & $31(1)$ & $32(1)$ & $31(1)$ & $0(1)$ & $8(1)$ & $-2(1)$ \\
\hline $\mathrm{C} 24$ & $26(1)$ & $30(1)$ & $38(1)$ & $-2(1)$ & $8(1)$ & $-3(1)$ \\
\hline $\mathrm{C} 25$ & $44(1)$ & $32(1)$ & $43(1)$ & $-1(1)$ & $9(1)$ & $-4(1)$ \\
\hline
\end{tabular}




\begin{tabular}{|c|c|c|c|c|c|c|}
\hline $\mathrm{C} 26$ & $55(1)$ & $33(1)$ & $36(1)$ & $-7(1)$ & $19(1)$ & $-10(1)$ \\
\hline C28 & $44(1)$ & $32(1)$ & $33(1)$ & $-2(1)$ & $15(1)$ & $-6(1)$ \\
\hline C29 & $45(1)$ & $45(1)$ & $65(1)$ & $0(1)$ & $5(1)$ & $2(1)$ \\
\hline $\mathrm{C} 30$ & $41(1)$ & $30(1)$ & $43(1)$ & $-2(1)$ & $13(1)$ & $-6(1)$ \\
\hline C31 & $57(4)$ & $46(7)$ & $54(2)$ & $1(1)$ & $31(3)$ & $0(4)$ \\
\hline C32 & $66(5)$ & $62(4)$ & $69(5)$ & $8(5)$ & $40(4)$ & $-2(4)$ \\
\hline C33 & $47(3)$ & $61(2)$ & $97(6)$ & $-12(5)$ & $33(4)$ & $-10(2)$ \\
\hline C34 & $41(1)$ & $64(2)$ & $88(6)$ & $4(5)$ & $19(4)$ & $-4(1)$ \\
\hline C35 & $38(2)$ & $52(4)$ & $56(4)$ & $1(4)$ & $12(2)$ & $-2(2)$ \\
\hline C30' & $41(1)$ & $30(1)$ & $43(1)$ & $-2(1)$ & $13(1)$ & $-6(1)$ \\
\hline C31' & $57(4)$ & $46(7)$ & $54(2)$ & $1(1)$ & $31(3)$ & $0(4)$ \\
\hline C32' & $66(5)$ & $62(4)$ & $69(5)$ & $8(5)$ & $40(4)$ & $-2(4)$ \\
\hline C33' & $47(3)$ & $61(2)$ & $97(6)$ & $-12(5)$ & $33(4)$ & $-10(2)$ \\
\hline C34' & $41(1)$ & $64(2)$ & $88(6)$ & $4(5)$ & $19(4)$ & $-4(1)$ \\
\hline C35' & $38(2)$ & $52(4)$ & $56(4)$ & $1(4)$ & $12(2)$ & $-2(2)$ \\
\hline $\mathrm{C} 27$ & $31(4)$ & $31(1)$ & $38(1)$ & $-4(1)$ & $17(2)$ & $-4(2)$ \\
\hline C36 & $70(3)$ & $34(1)$ & $36(2)$ & $0(1)$ & $18(2)$ & $-10(2)$ \\
\hline C37 & $62(2)$ & $26(2)$ & $34(1)$ & $1(1)$ & $17(1)$ & $-9(2)$ \\
\hline C38 & $68(3)$ & $41(2)$ & $51(3)$ & $-6(2)$ & $19(2)$ & $-4(2)$ \\
\hline C39 & $86(3)$ & $49(2)$ & $44(2)$ & $-11(1)$ & $13(2)$ & $-15(2)$ \\
\hline $\mathrm{C} 40$ & $118(4)$ & $40(2)$ & $61(3)$ & $-11(2)$ & $49(3)$ & $-8(3)$ \\
\hline $\mathrm{C} 41$ & $98(4)$ & $46(2)$ & $74(3)$ & $-4(2)$ & $46(3)$ & $5(2)$ \\
\hline $\mathrm{C} 42$ & $67(2)$ & $38(2)$ & $56(3)$ & $1(2)$ & $28(3)$ & $1(2)$ \\
\hline $\mathrm{C} 27^{\prime}$ & $31(4)$ & $31(1)$ & $38(1)$ & $-4(1)$ & $17(2)$ & $-4(2)$ \\
\hline C36' & $70(3)$ & $34(1)$ & $36(2)$ & $0(1)$ & $18(2)$ & $-10(2)$ \\
\hline C $37^{\prime}$ & $62(2)$ & $26(2)$ & $34(1)$ & $1(1)$ & $17(1)$ & $-9(2)$ \\
\hline C $38^{\prime}$ & $68(3)$ & $41(2)$ & $51(3)$ & $-6(2)$ & $19(2)$ & $-4(2)$ \\
\hline C39' & $86(3)$ & $49(2)$ & $44(2)$ & $-11(1)$ & $13(2)$ & $-15(2)$ \\
\hline $\mathrm{C} 40^{\prime}$ & $118(4)$ & $40(2)$ & $61(3)$ & $-11(2)$ & $49(3)$ & $-8(3)$ \\
\hline C41' & $98(4)$ & $46(2)$ & $74(3)$ & $-4(2)$ & $46(3)$ & $5(2)$ \\
\hline $\mathrm{C} 42^{\prime}$ & $67(2)$ & $38(2)$ & $56(3)$ & $1(2)$ & $28(3)$ & $1(2)$ \\
\hline
\end{tabular}


Table 5. Hydrogen coordinates $\left(\times 10^{4}\right)$ and isotropic displacement parameters $\left(\AA^{2} \times 10^{3}\right)$ for 03139 .

\begin{tabular}{|c|c|c|c|c|}
\hline & $\mathrm{x}$ & $\mathrm{y}$ & $\mathrm{z}$ & $\mathrm{U}(\mathrm{eq})$ \\
\hline $\mathrm{H} 1 \mathrm{~N}$ & $3169(14)$ & $2745(7)$ & $-1205(13)$ & 42 \\
\hline $\mathrm{H} 4 \mathrm{~A}$ & 2915 & 1941 & 352 & 43 \\
\hline H4B & 4280 & 2085 & 557 & 43 \\
\hline $\mathrm{H} 5 \mathrm{~A}$ & 2726 & 4005 & 1500 & 35 \\
\hline H8A & 3871 & 3635 & -2003 & 56 \\
\hline H8B & 4432 & 3885 & -826 & 56 \\
\hline $\mathrm{H} 8 \mathrm{C}$ & 3523 & 4269 & -1682 & 56 \\
\hline H10A & 2102 & 3975 & -3113 & 55 \\
\hline H11A & 380 & 3835 & -4435 & 77 \\
\hline $\mathrm{H} 12 \mathrm{~A}$ & -1149 & 3338 & -4055 & 77 \\
\hline H13A & -999 & 2998 & -2331 & 69 \\
\hline H14A & 717 & 3141 & -989 & 53 \\
\hline H15A & 2465 & 4749 & -869 & 56 \\
\hline H15B & 1238 & 4547 & -704 & 56 \\
\hline H17A & 659 & 4846 & 880 & 50 \\
\hline H18A & 893 & 5492 & 2312 & 57 \\
\hline H19A & 2620 & 6004 & 2907 & 60 \\
\hline $\mathrm{H} 20 \mathrm{~A}$ & 4132 & 5855 & 2095 & 59 \\
\hline $\mathrm{H} 21 \mathrm{~A}$ & 3895 & 5215 & 662 & 53 \\
\hline $\mathrm{H} 2 \mathrm{~N}$ & $6492(15)$ & $2787(8)$ & $2099(14)$ & 48 \\
\hline $\mathrm{H} 25 \mathrm{~A}$ & 5453 & 2116 & 3391 & 48 \\
\hline $\mathrm{H} 25 \mathrm{~B}$ & 6825 & 1976 & 3752 & 48 \\
\hline $\mathrm{H} 26 \mathrm{~A}$ & 7050 & 4034 & 5030 & 48 \\
\hline H29A & 5814 & 3635 & 944 & 80 \\
\hline H29B & 6216 & 4279 & 1341 & 80 \\
\hline $\mathrm{H} 29 \mathrm{C}$ & 5251 & 3953 & 1793 & 80 \\
\hline H31A & 7755 & 3803 & 624 & 59 \\
\hline $\mathrm{H} 32 \mathrm{~A}$ & 9668 & 3650 & 506 & 74 \\
\hline H33A & 11106 & 3365 & 1981 & 79 \\
\hline H34A & 10669 & 3202 & 3620 & 77 \\
\hline
\end{tabular}




\begin{tabular}{lrrrr} 
H35A & 8801 & 3393 & 3790 & 58 \\
H31B & 7472 & 3918 & 692 & 59 \\
H32B & 9169 & 3770 & 104 & 74 \\
H33B & 10791 & 3344 & 1276 & 79 \\
H34B & 10786 & 3145 & 3032 & 77 \\
H35B & 9100 & 3264 & 3589 & 58 \\
H36A & 7704 & 4728 & 2945 & 55 \\
H36B & 6327 & 4818 & 2670 & 55 \\
H38 & 8900 & 4930 & 4796 & 63 \\
H39 & 9103 & 5546 & 6316 & 72 \\
H40 & 7455 & 6044 & 6568 & 82 \\
H41 & 5691 & 5889 & 5413 & 82 \\
H42 & 5456 & 5280 & 3964 & 62 \\
H36C & 8528 & 4593 & 3750 & 55 \\
H36D & 7438 & 4750 & 2777 & 55 \\
H38' & 8744 & 5080 & 5422 & 63 \\
H39' & 8023 & 5739 & 6533 & 72 \\
H40' & 6080 & 6041 & 5974 & 82 \\
H41' & 4941 & 5766 & 4330 & 82 \\
H42' & 5634 & 5120 & 3265 & 62 \\
& & & & \\
\hline
\end{tabular}


Table 6. Torsion angles $\left[{ }^{\circ}\right]$ for 03139 .

\begin{tabular}{|c|c|c|c|}
\hline $\mathrm{C} 4-\mathrm{O} 1-\mathrm{C} 1-\mathrm{O} 2$ & $178.13(14)$ & C14-C9-C10-C11 & $-0.9(3)$ \\
\hline $\mathrm{C} 4-\mathrm{O} 1-\mathrm{C} 1-\mathrm{C} 2$ & $-1.77(17)$ & C7-C9-C10-C11 & $176.14(16)$ \\
\hline $\mathrm{O} 2-\mathrm{C} 1-\mathrm{C} 2-\mathrm{C} 3$ & $-179.45(17)$ & C9-C10-C11-C12 & $-0.2(3)$ \\
\hline $\mathrm{O} 1-\mathrm{C} 1-\mathrm{C} 2-\mathrm{C} 3$ & $0.43(17)$ & $\mathrm{C} 10-\mathrm{C} 11-\mathrm{C} 12-\mathrm{C} 13$ & $1.0(3)$ \\
\hline $\mathrm{O} 2-\mathrm{C} 1-\mathrm{C} 2-\mathrm{C} 5$ & $-3.6(3)$ & C11-C12-C13-C14 & $-0.8(3)$ \\
\hline $\mathrm{O} 1-\mathrm{C} 1-\mathrm{C} 2-\mathrm{C} 5$ & $176.31(14)$ & C10-C9-C14-C13 & $1.2(2)$ \\
\hline C7-N1-C3-C2 & $-0.6(2)$ & C7-C9-C14-C13 & $-176.02(14)$ \\
\hline C7-N1-C3-C4 & $177.19(14)$ & C12-C13-C14-C9 & $-0.3(3)$ \\
\hline $\mathrm{C} 1-\mathrm{C} 2-\mathrm{C} 3-\mathrm{N} 1$ & $179.17(14)$ & C5-C6-C15-C16 & $17.6(2)$ \\
\hline $\mathrm{C} 5-\mathrm{C} 2-\mathrm{C} 3-\mathrm{N} 1$ & $2.7(2)$ & C7-C6-C15-C16 & $-162.86(14)$ \\
\hline $\mathrm{C} 1-\mathrm{C} 2-\mathrm{C} 3-\mathrm{C} 4$ & $1.03(17)$ & C6-C15-C16-C17 & $-95.83(19)$ \\
\hline C5-C2-C3-C4 & $-175.48(13)$ & C6-C15-C16-C21 & $83.9(2)$ \\
\hline $\mathrm{C} 1-\mathrm{O} 1-\mathrm{C} 4-\mathrm{C} 3$ & $2.25(15)$ & C21-C16-C17-C18 & $2.2(2)$ \\
\hline N1-C3-C4-O1 & $179.90(14)$ & $\mathrm{C} 15-\mathrm{C} 16-\mathrm{C} 17-\mathrm{C} 18$ & $-178.10(15)$ \\
\hline $\mathrm{C} 2-\mathrm{C} 3-\mathrm{C} 4-\mathrm{O} 1$ & $-2.01(16)$ & C16-C17-C18-C19 & $-0.8(3)$ \\
\hline $\mathrm{C} 3-\mathrm{C} 2-\mathrm{C} 5-\mathrm{C} 6$ & $0.0(2)$ & C17-C18-C19-C20 & $-0.9(3)$ \\
\hline $\mathrm{C} 1-\mathrm{C} 2-\mathrm{C} 5-\mathrm{C} 6$ & $-175.47(16)$ & $\mathrm{C} 18-\mathrm{C} 19-\mathrm{C} 20-\mathrm{C} 21$ & $1.1(3)$ \\
\hline C2-C5-C6-C15 & $174.96(15)$ & $\mathrm{C} 19-\mathrm{C} 20-\mathrm{C} 21-\mathrm{C} 16$ & $0.4(3)$ \\
\hline $\mathrm{C} 2-\mathrm{C} 5-\mathrm{C} 6-\mathrm{C} 7$ & $-4.5(2)$ & $\mathrm{C} 17-\mathrm{C} 16-\mathrm{C} 21-\mathrm{C} 20$ & $-2.0(2)$ \\
\hline C3-N1-C7-C8 & $115.42(15)$ & $\mathrm{C} 15-\mathrm{C} 16-\mathrm{C} 21-\mathrm{C} 20$ & $178.29(15)$ \\
\hline C3-N1-C7-C9 & $-123.91(14)$ & $\mathrm{C} 25-\mathrm{O} 3-\mathrm{C} 22-\mathrm{O} 4$ & $-178.74(14)$ \\
\hline C3-N1-C7-C6 & $-3.36(19)$ & $\mathrm{C} 25-\mathrm{O} 3-\mathrm{C} 22-\mathrm{C} 23$ & $1.71(17)$ \\
\hline C5-C6-C7-N1 & $5.9(2)$ & $\mathrm{O} 4-\mathrm{C} 22-\mathrm{C} 23-\mathrm{C} 24$ & $179.59(17)$ \\
\hline C15-C6-C7-N1 & $-173.58(14)$ & $\mathrm{O} 3-\mathrm{C} 22-\mathrm{C} 23-\mathrm{C} 24$ & $-0.95(17)$ \\
\hline C5-C6-C7-C8 & $-111.39(16)$ & $\mathrm{O} 4-\mathrm{C} 22-\mathrm{C} 23-\mathrm{C} 26$ & $-0.2(3)$ \\
\hline $\mathrm{C} 15-\mathrm{C} 6-\mathrm{C} 7-\mathrm{C} 8$ & $69.09(17)$ & $\mathrm{O} 3-\mathrm{C} 22-\mathrm{C} 23-\mathrm{C} 26$ & $179.21(16)$ \\
\hline C5-C6-C7-C9 & $123.32(15)$ & C28-N2-C24-C23 & $5.6(2)$ \\
\hline C15-C6-C7-C9 & $-56.20(17)$ & C28-N2-C24-C25 & $-174.77(14)$ \\
\hline N1-C7-C9-C10 & $-120.31(16)$ & $\mathrm{C} 22-\mathrm{C} 23-\mathrm{C} 24-\mathrm{N} 2$ & $179.53(14)$ \\
\hline C8-C7-C9-C10 & $-3.7(2)$ & $\mathrm{C} 26-\mathrm{C} 23-\mathrm{C} 24-\mathrm{N} 2$ & $-0.6(2)$ \\
\hline C6-C7-C9-C10 & $119.51(16)$ & $\mathrm{C} 22-\mathrm{C} 23-\mathrm{C} 24-\mathrm{C} 25$ & $-0.16(17)$ \\
\hline N1-C7-C9-C14 & $56.76(17)$ & $\mathrm{C} 26-\mathrm{C} 23-\mathrm{C} 24-\mathrm{C} 25$ & $179.70(14)$ \\
\hline C8-C7-C9-C14 & $173.39(13)$ & $\mathrm{C} 22-\mathrm{O} 3-\mathrm{C} 25-\mathrm{C} 24$ & $-1.71(16)$ \\
\hline С6-C7-C9-C14 & $-63.42(17)$ & $\mathrm{N} 2-\mathrm{C} 24-\mathrm{C} 25-\mathrm{O} 3$ & $-178.54(14)$ \\
\hline
\end{tabular}




\begin{tabular}{|c|c|c|c|}
\hline $\mathrm{C} 23-\mathrm{C} 24-\mathrm{C} 25-\mathrm{O} 3$ & $1.14(17)$ & C30-C28-C27-C36 & $68.1(5)$ \\
\hline C24-C23-C26-C27' & $-13.6(4)$ & C29-C28-C27-C36 & $-56.9(5)$ \\
\hline $\mathrm{C} 22-\mathrm{C} 23-\mathrm{C} 26-\mathrm{C} 27^{\prime}$ & $166.2(4)$ & C27'-C28-C27-C36 & $95.6(16)$ \\
\hline $\mathrm{C} 24-\mathrm{C} 23-\mathrm{C} 26-\mathrm{C} 27$ & $7.2(4)$ & C26-C27-C36-C37 & $-1.9(9)$ \\
\hline C22-C23-C26-C27 & $-173.0(4)$ & $\mathrm{C} 28-\mathrm{C} 27-\mathrm{C} 36-\mathrm{C} 37$ & $-170.4(6)$ \\
\hline C24-N2-C28-C30 & $113.56(16)$ & C27-C36-C37-C42 & $-95(3)$ \\
\hline C24-N2-C28-C29 & $-125.60(16)$ & C27-C36-C37-C38 & $85(3)$ \\
\hline C24-N2-C28-C27' & $2.3(4)$ & C42-C37-C38-C39 & $-2(4)$ \\
\hline C24-N2-C28-C27 & $-14.4(4)$ & C36-C37-C38-C39 & $178.3(18)$ \\
\hline N2-C28-C30-C31 & $107(2)$ & C37-C38-C39-C40 & $-1(2)$ \\
\hline C29-C28-C30-C31 & $-9(2)$ & C38-C39-C40-C41 & $2.0(10)$ \\
\hline $\mathrm{C} 27^{\prime}-\mathrm{C} 28-\mathrm{C} 30-\mathrm{C} 31$ & $-136(2)$ & $\mathrm{C} 39-\mathrm{C} 40-\mathrm{C} 41-\mathrm{C} 42$ & $-1.1(12)$ \\
\hline $\mathrm{C} 27-\mathrm{C} 28-\mathrm{C} 30-\mathrm{C} 31$ & $-129(2)$ & $\mathrm{C} 40-\mathrm{C} 41-\mathrm{C} 42-\mathrm{C} 37$ & $-1(2)$ \\
\hline N2-C28-C30-C35 & $-70.4(10)$ & C38-C37-C42-C41 & $3(4)$ \\
\hline C29-C28-C30-C35 & $173.2(10)$ & C36-C37-C42-C41 & $-177.4(18)$ \\
\hline C27'-C28-C30-C35 & $46.0(10)$ & C27-C26-C27'-C36' & $91.7(17)$ \\
\hline C27-C28-C30-C35 & $53.3(10)$ & C23-C26-C27'-C36' & $-170.5(4)$ \\
\hline C35-C30-C31-C32 & $0(4)$ & $\mathrm{C} 27-\mathrm{C} 26-\mathrm{C} 27^{\prime}-\mathrm{C} 28$ & $-75.6(15)$ \\
\hline C28-C30-C31-C32 & $-177.0(17)$ & $\mathrm{C} 23-\mathrm{C} 26-\mathrm{C} 27^{\prime}-\mathrm{C} 28$ & $22.2(7)$ \\
\hline C30-C31-C32-C33 & $0(4)$ & N2-C28-C27'-C26 & $-16.3(7)$ \\
\hline C31-C32-C33-C34 & $0(3)$ & C30-C28-C27'-C26 & $-129.6(5)$ \\
\hline C32-C33-C34-C35 & $-2(2)$ & C29-C28-C27'-C26 & $105.6(6)$ \\
\hline C33-C34-C35-C30 & $3(2)$ & $\mathrm{C} 27-\mathrm{C} 28-\mathrm{C} 27^{\prime}-\mathrm{C} 26$ & $75.3(15)$ \\
\hline C31-C30-C35-C34 & $-2(3)$ & N2-C28-C27'-C36' & $176.0(4)$ \\
\hline C28-C30-C35-C34 & $175.8(14)$ & $\mathrm{C} 30-\mathrm{C} 28-\mathrm{C} 27^{\prime}-\mathrm{C} 36^{\prime}$ & $62.7(6)$ \\
\hline C31'-C32'-C33'-C34' & $-3(3)$ & C29-C28-C27'-C36' & $-62.2(6)$ \\
\hline C32'-C33'-C34'-C35' & $4(2)$ & $\mathrm{C} 27-\mathrm{C} 28-\mathrm{C} 27^{\prime}-\mathrm{C} 36^{\prime}$ & $-92.4(16)$ \\
\hline C27'-C26-C27-C36 & $-93.0(17)$ & C26-C27'-C36'-C37' & $-24.9(11)$ \\
\hline $\mathrm{C} 23-\mathrm{C} 26-\mathrm{C} 27-\mathrm{C} 36$ & $174.5(4)$ & $\mathrm{C} 28-\mathrm{C} 27^{\prime}-\mathrm{C} 36^{\prime}-\mathrm{C} 37^{\prime}$ & $143.0(8)$ \\
\hline C27'-C26-C27-C28 & $74.5(15)$ & C27'-C36'-C37'-C42' & $-75(3)$ \\
\hline C23-C26-C27-C28 & $-18.0(7)$ & C27'-C36'-C37'-C38' & $105(3)$ \\
\hline N2-C28-C27-C26 & $20.7(6)$ & C42'-C37'-C38'-C39' & $1(4)$ \\
\hline C30-C28-C27-C26 & $-101.0(5)$ & C36'-C37'-C38'-C39' & $-178.7(19)$ \\
\hline C29-C28-C27-C26 & $133.9(5)$ & C37'-C38'-C39'-C40' & $0(2)$ \\
\hline C27'-C28-C27-C26 & $-73.5(15)$ & C38'-C39'-C40'-C41' & $-2.5(12)$ \\
\hline N2-C28-C27-C36 & $-170.2(4)$ & C39'-C40'-C41'-C42' & $2.9(11)$ \\
\hline
\end{tabular}


Symmetry transformations used to generate equivalent atoms: 
Table 7. Hydrogen bonds for 03139 [ $\AA$ and ${ }^{\circ}$ ].

\begin{tabular}{lllll}
\hline D-H...A & $\mathrm{d}(\mathrm{D}-\mathrm{H})$ & $\mathrm{d}(\mathrm{H} \ldots \mathrm{A})$ & $\mathrm{d}(\mathrm{D} \ldots \mathrm{A})$ & $<(\mathrm{DHA})$ \\
\hline N1-H1N...O2\#1 & & & & \\
N2-H2N...O4\#1 & $0.918(17)$ & $1.928(17)$ & $2.8257(17)$ & $165.5(15)$ \\
& $0.910(18)$ & $2.003(18)$ & $2.8860(17)$ & $163.1(16)$ \\
\hline
\end{tabular}

Symmetry transformations used to generate equivalent atoms:

$\# 1 \mathrm{x},-\mathrm{y}+1 / 2, \mathrm{z}-1 / 2$ 